tienten, die ihre Antidiabetika komplett absetzen konnten!“

\section{Motivation mit einem interaktiven Bewegungs- spiel}

In einer dritten Studie mit 220 körperlich untätigen Diabetikern war es der Gruppe um Prof. Martin durch die bloße Bereitstellung einer Wii-Spielekonsole mit dem interaktiven Bewegungsspiel Wii Fit Plus gelungen, die Couch-Potatoes zu mobilisieren, eine Gewichtsinduktion und eine $\mathrm{HbA}_{1 \mathrm{c}}$-Absenkung $\mathrm{zu}$ induzieren sowie die Lebensqualität der Patienten spürbar zu erhöhen.

\section{Neu: „Deutsches Institut für Tele- medizin und Gesundheitsförderung"} Martin ist überzeugt, dass sich in der Prävention und Therapie des Diabetes mellitus viel erreichen lässt, wenn alle diese Maßnahmen kombiniert werden. „In Einzelfällen ist der Diabetes dann sogar reversibel“, so Martin gegenüber MMW.

Um die Maßnahmen in strukturierter Form einem breiten Patientenkreis zugänglich zu machen, hat er ein „Deutsches Institut für Telemedizin und Gesundheitsförderung “ gegründet. Der erste Auftrag von der Zentralversicherung liegt schon vor, wo sich Patienten ein iPhone erarbeiten können, wenn sie ihren Aktivitätsgrad nachhaltig erhöhen. „Wir müssen deutlich mehr Augenmerk auf diese allgemeinpräventiven Maßnahmen legen und damit die Ursachen des Diabetes angehen“, so Martin, „denn wir werden angesichts der $\mathrm{Zu}$ nahme der Patientenzahlen die nötigen Medikamente langfristig nicht mehr finanzieren können."

DR. MED. DIRK EINECKE .

- Quelle: American Diabetes Association, 73rd scientific sessions, 21.-25.6.2013 in Chicago

Typ-2-Diabetiker

\title{
Zwei größere Mahlzeiten besser als viele kleine
}

beiden Gruppen gleich. Nach zwölf Wochen erfolgte der Wechsel in die jeweils andere Gruppe.

\section{Adipositas: Diabetes- Risikofaktor Nr. 1.}

Für Patienten mit Typ-2-Diabetes ist es anscheinend günstiger, nur zwei Mahlzeiten pro Tag zu sich zu nehmen, als die gleiche Kalorienmenge nach dem oft empfohlenen Häppchen-Prinzip auf sechs kleine Mahlzeiten zu verteilen.

— Frühstücken wie ein Kaiser, Mittagessen wie ein König, Abendessen wie ein Bettler - diese alte Ernährungsregel scheint zumindest bei übergewichtigen Patienten mit Typ-2-Diabetes einer wissenschaftlichen Überprüfung durchaus standzuhalten.

\section{Zwei versus sechs Mahlzeiten}

Vorgenommen hat den Test eine Forschergruppe um Dr. Hana Kahleova aus Prag. In ihrer randomisierten Crossover-Studie haben die tschechischen Untersucher 54 mit oralen Antidiabetika behandelte Typ-2-Diabetiker auf eine kalorienreduzierte Diät (-500 kcal/Tag) gesetzt. Der Body-Mass-Index (BMI) der Teilnehmer lag im Schnitt bei 32,6, ihr Alter bei knapp 60 Jahren und der $\mathrm{HbA}_{1 \mathrm{c}}$-Wert bei 7,2\%

In Gruppe A gönnten sich die $\mathrm{Pa}$ tienten zwölf Wochen lang im Tagesverlauf bis zum Abend insgesamt sechs kleinere Mahlzeiten oder Snacks. Bei gleicher Kalorienbeschränkung gaben sich die der Gruppe B zugeteilten Diabetiker in dieser Zeit mit einem jeweils üppigeren Frühstück und Mittagessen zufrieden.

Der Anteil an Kohlenhydraten, Fett und Proteinen in der Nahrung war in

\section{Bessere Ergebnisse mit dem Zwei-Mahlzeiten-Konzept}

Das Resultat: Körpergewicht und BMI schmolzen in beiden zwölfwöchigen Phasen, jedoch signifikant stärker in der „Zwei-Mahlzeiten-Phase“.

Unter beiden hypokalorischen Diäten verringerte sich zudem der Fettgehalt in den Leberzellen. Auch diese Reduktion war bei Beschränkung auf nur zwei Mahlzeiten pro Tag deutlich ausgeprägter, berichtete Kahleova beim Kongress der American Diabetes Association (ADA) in Chicago.

Ein erhöhter Fettgehalt in Hepatozyten beeinträchtigt unter anderem die Fähigkeit von Insulin, die hepatische Glukoseproduktion zu hemmen.

Die Abnahme des Leberfetts korrelierte mit einer Zunahme der Insulinsensitivität, wobei auch diese Zunahme in der Phase mit nur zwei Mahlzeiten signifikant ausgeprägter war.

Peter Overbeck

- Quelle: Posterpräsentation Abstract 163-OR bei der American Diabetes Association, 73rd scientific sessions, 21.-25.6.2013 in Chicago

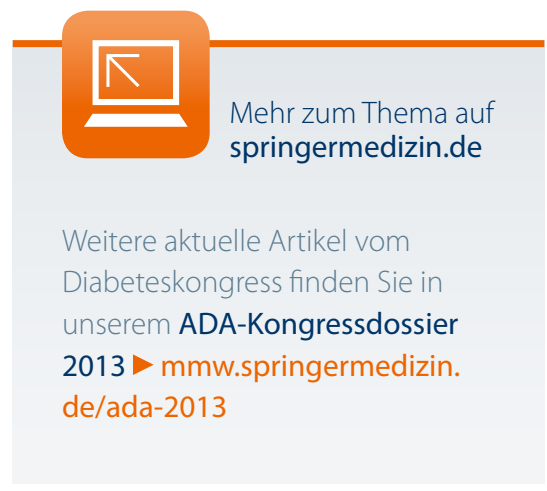

